\title{
THE ASSESSMENT OF INDUCED CONTINUOUS VARIATION IN PURE-BREEDING LINES FOLLOWING SELFING
}

\author{
D. S. VIRK, J. L. JINKS and H. S. POONI \\ Department of Genetics, University of Birmingham, Birmingham B15 2TT
}

Received: 15.vi.77

\begin{abstract}
SUMMARY
A simple method is described for assessing the genetical and environmental components of the continuous variation observed in a hierarchically structured selfing series which has been initiated from a pure-breeding line followiag mutagenic treatment. The simplicity of the method and its independence of any assumptions about the distribution of mutational events arises from the omission of all rank 0 statistics from the analysis. By proceeding in this way, from the $\mathbf{M}_{3}$ generation onwards sufficient statistics are generated within a single generation to provide estimates of all the components of variation. The estimates of the genetical components provide direct comparisons of the additive and dominance gene action and predictions of the long-term outcome of the inbreeding programme. Alternative methods, to accommodate statistics of rank 0 and the generation means, both of which were previously discussed by Aastveit and Gaul (1967), are also presented.

The new procedures are illustrated by the analysis of the data of Borojevic and Borojević (1969) on plant height and kernel number in the $\mathbf{M}_{5}, \mathbf{M}_{6}$ and $\mathbf{M}_{7}$ generations of two pure-breeding varieties of wheat which had been subjected to gamma ray treatments.
\end{abstract}

\section{INTRODUGTION}

VARIATION generated following mutagenic treatment is often classified into macro- and micro-mutations although this categorisation is only arbitrary (Gaul, 1965). So long as we maintain this classification the macro-mutations are those changes which give rise to discontinuous variation and are therefore easily isolated in early generations while micro-mutations result in continuous variation where segregation persists through advanced generations. The two types of induced variation therefore require different methods of investigation.

The majority of agronomically important plant characters are quantitatively inherited. Discrete classes can only rarely be recognised and classical Mendelian methods for genetical analysis are impractical though not impossible. The characters that display continuous variation are in general controlled by many genes showing Mendelian inheritance but subject to environmental modification. The creation of such variation for selection to act upon is very important for crop improvement and this has long been accomplished through hybridisation. But more recently, variation for quantitative characters, which has been induced by mutagenic treatments, has been widely demonstrated to be the result of polygenic differences arising from micro-mutations. The biometrical approach which uses statistical quantities such as means, variances and covariances to partition the variation into components which measure the different types of gene action has reached a high level of sophistication for evaluating the variation 
created by hybridisation, but with rare exceptions (Kao, Hu, Chang and Oka, 1960; Aastveit and Gaul, 1967) little development has taken place for partitioning polygenic variation induced by mutagenesis. In this paper we re-examine and modify existing biometrical genetical procedures for the assessment of induced polygenic variation in self-pollinating species and we illustrate a new approach by re-analysing the data of Borojević and Borojević (1969) on gamma ray irradiated pure-breeding wheat populations.

\section{Existing MODELS AND ASSUMptions}

The difficulty is not in deriving biometrical genetical expectations but of equating them to the kind of data that are generated without having to make untestable assumptions or alternatively having to restrict the analysis to only part of the data. The problem of untestable assumptions arises immediately one attempts to specify the frequency of genotypes in respect of a single locus in the generation following mutagenic treatment $\left(M_{1}\right)$. Kao et al. (1960) assume that allele $A$ mutates to $a$ at the rate $p$ when an $A A$ individual is treated. They then define the $\mathrm{M}_{1}$ population as consisting entirely of $(1-p) A A$ and $p A a$ plants. They omit the possibility that both $A$ alleles may mutate to the same or different mutant alleles. Aastveit and Gaul (1967) on the other hand, avoid the necessity of making this or an alternative assumption by using the $\mathrm{M}_{2}$ generation derived by selfing the $\mathrm{M}_{1}$ as the starting point of their analysis. They then allow the three kinds of individuals, in respect of a single gene locus, that is, $A A, A a$ and $a a$ to arise in the $\mathrm{M}_{2}$ with arbitrary frequencies $p, q$ and $r$. On the other hand, to produce a set of equations which are soluble when applied to data they concentrate on statistics of a single rank in successive generations rather than on different ranks in the same generation (see Mather and Jinks, 1971, page 132).

It is clearly advantageous to avoid any analysis whose outcome is dependent upon assumptions about the genotypic frequencies generated in the $M_{1}$ generation. It is also desirable to be able to use data from more than one rank since the natural hierarchical structure of selfing programmes can yield many ranks per generation in later generations and of course different ranks of the same generation are raised in the same season thus avoiding the confounding effects of seasonal differences.

One way of achieving both of these aims is to confine our attention to the variation that arises within the progenies of individual $M_{1}$ plants, treating different $M_{1}$ plants more or less as replicates. Each $M_{1}$ plant can then be regarded as an $F_{1}$ between hypothetical parents which in successive generations of selfing yield progenies whose properties can be completely specified since $\mathrm{M}_{2}=\mathrm{F}_{2}, \mathrm{M}_{3}=\mathrm{F}_{3}$ etc. whose means, variances and covariances are well known (Mather and Jinks, 1971). In this way we are analysing the contribution to the variation of loci which have become heterozygous in the $F_{1}$ as a result of the mutagenic treatment. To complete the expectations and the analysis we merely have to average the corresponding expectations and observed statistics over the independent progenies derived from different $M_{1}$ plants. But while the latter can be regarded as independent replicates, any assumptions about the distribution of gene and genotype frequencies in the $M_{1}$ generation will affect these expectations. In practice, however, the number and kind of parameters and their co- 
efficients in the expectations are independent of any such assumption; these affect only the composition and hence the precise definition of the parameters themselves.

\section{Theoretical Expegtations}

Following mutagenic treatment of a pure-breeding line any one $M_{1}$ plant will be heterozygous at a number of loci, $k$, either for the original allele $A$ and a mutant allele $a$ or for two mutant alleles $a_{1}$ and $a_{2}$. On. selfing each of these $k$ loci will segregate with the expectations typical of an $\mathrm{F}_{2}$ so that the contribution of this segregation to the expected variance $V_{M 2}$ will be

$$
V_{M 2}=V_{F 2}=\frac{1}{2} \sum_{i=1}^{k} d_{i}^{2}+\frac{1}{4} \sum_{i=1}^{k} h_{i}^{2} \quad \text { (Mather and Jinks, 1971) }
$$

If we now continue selfing and at the same time retain a hierarchical structure the contribution of the segregation to the variance of $r$ th rank in the $n$th generation $V_{r M_{n}}$ is given by the general formula of Mather and Jinks. (1971).

where

$$
V_{r M n}=V_{r F n}=\left(\frac{1}{2}\right)^{r} D+\left(\frac{1}{2}\right)^{2 n-r-1} H
$$

$$
D=\sum_{i=1}^{k} d_{i}^{2} \text { and } H=\sum_{i=1}^{k} h_{i}^{2}
$$

The corresponding parent offspring covariance $W_{r M_{n}\left(n^{-1}\right)}$ is given by

$$
W_{r M n(n-1)}=W_{r F n(n-1)}=\left(\frac{1}{2}\right)^{r} D+\left(\frac{1}{2}\right)^{2 n-r-2} H
$$

These formulae apply directly to the variances and covariances derived from the progenies of any one $M_{1}$ plant. To extend them to the average variances and covariances obtained from the progenies of all $M_{1}$ plants we merely have to modify the definition of $D$ and $H$. These necessary. modifications are the generalised forms of $D$ and $H$ given by Dickinson and Jinks (1956). Thus if we assume that in the $M_{1}$ generation the three kinds. of individuals $A A, A a$ and $a a$ in respect of any one locus arise with arbitrary frequencies $\alpha, \beta$, and $\gamma$ as a result of one of both $A$ alleles mutating to $a$, or vice versa, then summing over $k$ such loci only a proportion $\beta$ will be contributing to the variation defined by the general formulae and the: definitions of $D$ and $H$ become

$$
\sum_{i=1}^{k} 2 \beta_{i} d_{i}^{2} \text { and } \sum_{i=1}^{k} 2 \beta_{i} h_{i}^{2} \quad \text { respectively, where } \alpha_{i}+\beta_{i}+\gamma_{i}=1 \text {. }
$$

On the other hand, if we assume that $A$ mutates to $a$ with a frequency of $v$. and that mutation at each allele is independent so that $A A$ mutates to $a a$ with a frequency of $v^{2}$, then summing over $k$ such loci only a proportion $2 u v$ will be contributing to the variation defined by the general formulae and the definitions of $D$ and $H$ become

$$
\sum_{\substack{i=1 \\ 40 / 2-\mathrm{F}}}^{k} 4 u_{i} v_{i} d_{i}^{2} \text { and } \sum_{i=1}^{k} 4 u_{i} v_{i} h_{i}^{2} \quad \text { respectively, where } u_{i}=\left(1-v_{i}\right)
$$


Clearly, we can accommodate any assumption about gene and genotype frequency in the $\mathrm{M}_{1}$ by adjusting the coefficient of $d^{2}$ and $h^{2}$. Furthermore, the coefficient will always be twice the frequency of heterozygotes in the $M_{1}$ and it will be identical for $d^{2}$ and $h^{2}$. Thus the estimation of $D$ and $H$ and the relative values of the estimates is independent of any assumptions about gene and genotype frequencies in the $\mathbf{M}_{1}$ generation. Our general formulae, however, are based on a frequency of heterozygotes in the $F_{1}=M_{1}$ of one, whereas in our models it is $\beta$ or $2 u v$ and the maximum frequency that could be obtained, for example, with the random model $(u, v)$ is half when $u=v$. To put it another way, both the additive and dominance components of variation in our models are half of those expected from the general formulae if we use the general definitions of $D$ and $H$. To accommodate this we must halve the coefficient of $D$ and $H$ in the general formulae no matter which model we assume if we use these formulae for statistics averaged over the progenies of all $\mathrm{M}_{1}$ plants.

There are two ways of making this adjustment. We can maintain the convention that rank $\mathrm{l}(r=1)$ in the selfing series refers to the variation among $\mathrm{F}_{2}\left(\mathrm{M}_{2}\right)$ individuals; in which case we must substitute $(r+1)$ for $r$ in the general formulae. Or, we can recognise that in the special case we are considering the highest hierarchy (rank 1) is the variation among $F_{1}\left(M_{1}\right)$ individuals; in which case the highest rank specified by the general formula is not $r=1$ but $r=2$. The latter is simpler but the alternative has been adopted (see table 1) to avoid confusion with the conventional use of the general formulae and of the concept of rank.

TABLE 1

The genetical components of variances and parent-offspring covariances in the generations produced by selfing following mutagenic treatment of a pure-breeding line

\begin{tabular}{|c|c|c|c|c|c|}
\hline \multirow[b]{2}{*}{ Statistic } & \multicolumn{5}{|c|}{ Parameters $\uparrow$} \\
\hline & $D_{1}$ & $D$ & $H$ & $H_{1}$ & $F_{1}$ \\
\hline $\begin{array}{l}V_{O M n} \\
V_{\text {rMn }} \\
W_{O M n(n-1)} \\
W_{\text {rM } n(n-1)}\end{array}$ & $\begin{array}{l}\frac{1}{2} \\
0 \\
\frac{1}{2} \\
0\end{array}$ & $\begin{array}{c}0 \\
\left(\frac{1}{2}\right)^{r+1} \\
0 \\
\left(\frac{1}{2}\right)^{r+-1}\end{array}$ & $\begin{array}{l}\left(\frac{1}{2}\right)^{2 n-1} \\
\left(\frac{1}{2}\right)^{2 n-r} \\
\left(\frac{1}{4}\right)^{n-1} \\
\left(\frac{1}{2}\right)^{2 n r-1}\end{array}$ & $\begin{array}{c}-\left(\frac{1}{4}\right)^{n} \\
0 \\
-\left(\frac{1}{2}\right)^{2 n-1} \\
0\end{array}$ & $\begin{array}{c}-\left(\frac{1}{2}\right)^{n} \\
0 \\
-3\left(\frac{1}{2}\right)^{n+1} \\
0\end{array}$ \\
\hline
\end{tabular}

The expectations described so far are all based on statistics which describe the variation within the progenies derived from $M_{1}$ plants. We can, however, readily extend the expectations to statistics of rank 0 which describe the variation between $M_{1}$ plants. Indeed, the appropriate expectations are already in the literature (Dickinson and Jinks, 1956; Aastveit and Gaul, 1967). But to do so we must introduce two or three additional genetical parameters and the assumptions upon which they are based. Thus if we take the arbitrary genotype frequencies of $\alpha, \beta$ and $\gamma$ we must introduce the three parameters 
and

$$
\begin{aligned}
& D_{1}=\sum_{i=1}^{k} 2 Z_{i} d_{i}^{2} \\
& H_{1}=\sum_{i=1}^{k} 4 \beta_{i}^{2} h_{i}^{2}
\end{aligned}
$$

where

$$
F_{1}=\sum_{i=1}^{k} 4 \beta_{i}\left(\alpha_{i}-\gamma_{i}\right) d_{i} h_{i}
$$

$$
Z_{i}=\left[4 \alpha_{i} \gamma_{i}+\beta_{i}\left(1+\beta_{i}\right)\right] \text {. }
$$

If, however, we use the genotype frequencies $u^{2}, 2 u v$ and $v^{2}$ which assumes that alleles at the same locus mutate independently we need to introduce only two parameters.

and

$$
H_{1}=\sum_{i=1}^{k} 16 u_{i}^{2} v_{i}^{2} h_{i}^{2}
$$

$$
F_{1}=\sum_{i=1}^{k} 8 u_{i} v_{i}\left(u_{i}-v_{i}\right) d_{i} h_{i}
$$

since $D_{1}$ will then equal $D$.

The expected genetical contribution to the variances and covariances of all ranks for the general case are given in table 1. Before these can be used in practice the appropriate sampling errors and the environmental components must be added (Mather and Jinks, 1971). The complete specification requires five genetical components and at least one and usually two or more environmental components. By including both $D$ and $D_{1}$ in the specifications no assumption about the gene and genotype frequencies is required and we can always subsequently test the validity of the assumption that leads to $D=D_{1}$.

There are two strategies for reducing the number of genetical components to the level where they may be readily estimated. The first was proposed by Aastveit and Gaul (1967) who, by confining attention to rank 0 statistics, could omit $D$ and combine $H$ and $H_{1}$ into a single parameter with the composition $\left(H-\frac{1}{2} H_{1}\right)$. The alternative we are proposing is to omit rank 0 statistics and thereby remove the need for $D_{1}, H_{1}$ and $F_{1}$. The first obviously makes better use of the data from early generations but it requires one more statistic for estimating the additional parameter. The alternative makes much better use of data from later generations, has one less parameter and estimates parameters which are more simply interpreted in terms of gene action.

TABLE 2

The genetical expectations of the means of the generations produced by selfing following mutagenic treatment of a pure-breeding line

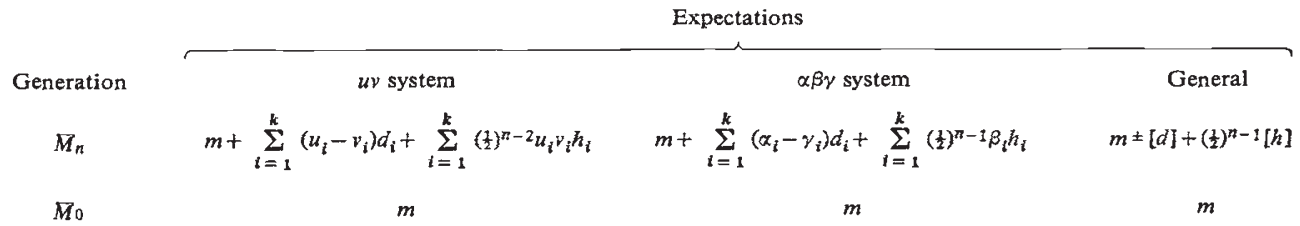


Aastveit and Gaul (1967) also gave expectations for the generation means $\left(\bar{M}_{n}\right)$ and in table 2 we present these expectations for the initial parental material, $n=0$, and for the general case. The parameter $m$ which normally defines the mid-parent value in a cross between two pure-breeding lines is here the mean of the untreated pure-breeding line which has undergone mutagenic treatment. If, therefore, the mutation rate is zero all the generation means have an expectation of $m$ only.

\section{ESTIMATION OF COMPONENTS}

Aastveit and Gaul (1967) have already described how the components may be estimated from rank 0 statistics and from generation means. We shall, therefore, confine our attention to the alternative approach. Given that untreated control material has been raised which has been derived from the pure-breeding line with exactly the same hierarchical structure as the material derived from the same line following the mutagenic treatment, estimates of all the appropriate environmental components of variation, whatever their number and kind, will be available. Any generation of treated material grown simultaneously with the controls that can provide two or more variances or covariances of rank $>1$ will, therefore, allow the genetical parameters and all necessary environmental parameters to be estimated. If more than the minimum of two such statistics are available weighted least squares estimates of the parameters may be obtained by iteration and the goodness of fit of the model tested by a $\chi^{2}$. The $M_{3}$ generation alone would, therefore, provide the statistics for a perfect fit solution while the $\mathrm{M}_{4}$ or any other later generation alone would provide weighted least squares estimates. From the $\mathrm{M}_{3}$ onwards we can obtain at least one parent-offspring covariance of rank $>1$. But while this adds to the statistics available for a least squares solution, covariances have the disadvantage of involving comparisons over seasons and are therefore subject to interactions of genotypes with seasonal differences.

By using generations as late as or later than the $M_{4}$ there is plenty of opportunity for identifying and eliminating from the data the contributions of macro-mutations for which alternative methods of analysis are available. On the other hand, the contribution that dominance makes to the variation or indeed to the means in these generations is so small (tables 1 and 2) that only the most sensitive experiment is likely to detect its presence and estimates of its magnitude are unlikely to be sufficiently precise to have any predictive value. Thus we lose the information that might have thrown light on the genetical architecture but we retain the information which is required to predict the properties of the pure-breeding lines that will eventually emerge from the selfing series (Jinks and Pooni, 1976).

The analysis of the generation means can proceed at two levels. The first is to test whether for any generation the mean of the progenies derived from the treated material differs from that of the corresponding progenies of the control material. If the deviation between the means is significant mutations have been induced. If it is not significant, however, it does not necessarily show that mutations have not occurred since the deviation results from asymmetrical or directional effects of the treatment. Thus the contributors to this deviation are (i) a difference in the frequency of increasing alleles $(A)$ which have mutated to decreasing alleles $(a)$ and of 
decreasing alleles $(a)$ which have mutated to increasing alleles $(A)$, and (ii) a difference in the frequency of mutations at loci for which the increasing allele is dominant and for which the decreasing allele is dominant. Should the directional effects (i) and (ii) be equal but opposite in sign there will again be no evidence from an analysis of generation means that any mutations have been induced.

If the deviations are significant we can then test whether an additive, dominance model will account for their relative magnitudes over a number of generations. This can be examined using the standard joint scaling test procedures (Mather and Jinks, 1971). From the signs of the estimates of the significant components of this model we can determine the net direction of the mutations. If $[d]$ is significantly positive the net direction is mutation of decreasing alleles to increasing alleles $(a \rightarrow A)$ while if negative it is of increasing alleles to decreasing alleles $(A \rightarrow a)$. Similarly if $[h]$ is significantly positive more increasing alleles are dominant than decreasing alleles while if negative it is the reverse.

\section{Examples}

We shall illustrate our new analysis based upon the exclusion of rank 0 statistics with data from experiment II of Borojević and Borojević (1969). This consists of the $M_{5}, M_{6}$ and $M_{7}$ generations of two gamma ray treated (10kr, $15 \mathrm{kr}$ and $20 \mathrm{kr}$ ) varieties (San Pastore and Bankut-1205) of Triticum vulgare. Two characters, plant height and kernel number, were recorded on all the progenies of the morphologically normal plants.

The structure of the experiment permits the recognition in each generation derived from the irradiated material and from the controls of all the hierarchies or ranks that are theoretically possible with the exception of rank 1 which is confounded with rank 0 . The mean squares corresponding with these hierarchies in an analysis of variance are tabulated by Borojević and Borojević (1969) in their tables XI to XXVI. The expectations of these mean squares in terms of the statistical components of variation are given in table 3 for the $M_{5}$ generation. We can arrive at their equivalence in terms of the genetical and environmental components of our model by substituting the special case of $n=5$ in the general formulae in table 1 . For $n=5$ there are four variances corresponding with $r=1$ to 4 and we are excluding $r=1$ from consideration. Taking the simplest variance $V_{4} M_{5}$ and applying our general formula (table 1) it has the expectation $\frac{1}{32} D+\frac{1}{64} H$ to which we must add the sampling error which is zero and the environmental component which is $E_{1}$ (Mather and Jinks, 1971). The expectation of the rank 4 item (i) Treated, in table 3 , the within family mean square $\left(\sigma_{4}^{2}\right)$, is therefore,

$$
V_{4 M 5}=\frac{1}{32} D+\frac{1}{64} H+E_{1}
$$

As a further example we can take the rank 3 variance $V_{3 M 5}$. From the general formula in table 1 this has the expectation $\frac{1}{16} D+\frac{1}{128} H$ to which we must add the sampling variance $\frac{1}{k} V_{4 M 5}$ and the environmental component $E_{2}$ (Mather and Jinks, 1971) to give

$$
V_{3 M 5}=\frac{1}{16} D+\frac{1}{128} H+E_{2}+\frac{1}{k}\left(\frac{1}{32} D+\frac{1}{64} H+E_{1}\right)
$$




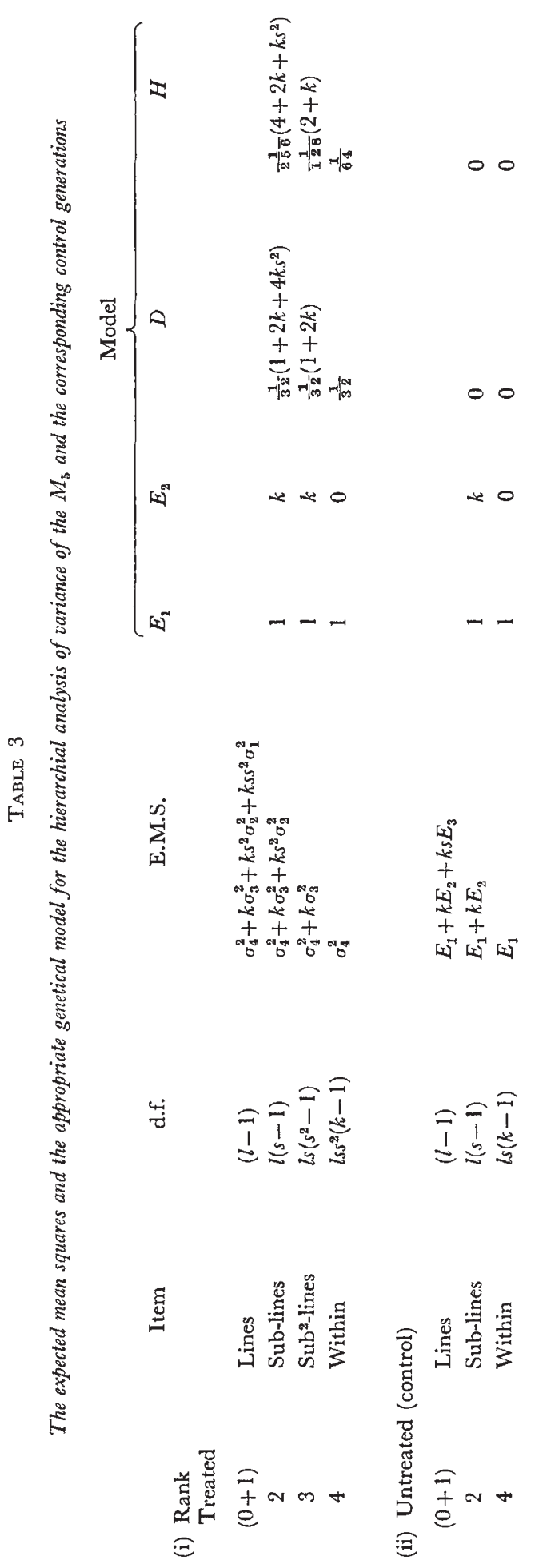


The expectation of the mean square for the rank 3 item (i) Treated, in table 3 , is then $k V_{3 M 5}$ where $k$ is the number of plants in a family.

In the same way we can arrive at the expectation of the rank 2 mean square,

$k s^{2} V_{2 M 5}=k s^{2}\left[\frac{1}{8} D+\frac{1}{256} H+\frac{1}{s^{2}}\left(\frac{1}{16} D+\frac{1}{128} H+E_{2}\right)+\frac{1}{k s^{2}}\left(\frac{1}{32} D+\frac{1}{64} H+E_{1}\right)\right]$

where $s^{2}$ is the number of sub-sub-lines in each $\mathbf{M}_{1}$ set in the hierarchy. The corresponding expectations for the $M_{6}$ and $M_{7}$ generations can be derived by simple extrapolation. By equating these expectations to the observed mean squares of Borojević and Borojević (1969) the components of the models were estimated.

The structure of the experiment does not demand more than two environmental parameters, $E_{1}$ and $E_{2}$. However, a number of higher ranking mean squares in the analyses of variance of the controls are significant and this requires the addition of a third and even a fourth environmental component of variation. These significances may be due to correlated environments at levels above that of the family or to residual heterozygosity in the pure-breeding varieties. But they must be accounted for by the model otherwise there is no way of separating out the variation induced by the treatment. When no simple model would fit the data the 10 per cent level of significance in the analysis of variance was used as a conservative guide in deciding whether or not these additional parameters should be added to the model. In general, however, parameters were retained in a model only if they were necessary for achieving a satisfactory fit with the data and if their estimates were significant $(P \gtrless 0.05)$.

The results of fitting the models are summarised in tables 4 and 5 . In some instances with variety Bankut-1205 (table 5) no model could be found which adequately accounted for the observations and these are omitted from the table. We can only assume that in these instances, additional sources of variation not allowed for in our models, such as epistasis, linkage and genotype $\times$ environment interactions are present at a significant level.

For many of the combinations in tables 4 and 5 models containing only environmental components were adequate. For these, there is no evidence that any genetical variation has been induced by the treatment. This result is not unexpected since reference to the hierarchical analyses of variance shows that for these combinations the mean squares for the treatment are no greater than the corresponding mean squares for the control. For the remaining combinations it was necessary to include the additive genetical component $D$ as well as the environmental components in the model to achieve an adequate fit with the data. But, as anticipated, the coefficients of the dominance component $H$ in the expectations were so small that no significant contributions to the variation from this source were detectable.

For seven of the 29 combinations included in tables 4 and 5 two alternative models $(a$ and $b$ ) are presented. In each case there is some ambiguity about which is the better. For kernel number in Bankut-1205 two alternative models fit the $M_{7}$ generation at all three levels of irradiation. The ambiguity arises solely from the $E_{2}$ component. The models omitting $E_{2}$ are adequate and lead to a non-significant $\chi^{2}$. Furthermore, the models including $E_{2}$ give non-significant estimates for this component. On our normal criteria therefore, the models excluding $E_{2}$ would be preferred and 
TABLE 4

Model fitting for the variation in plant height and kernel number in the $M_{5}, M_{6}$ and $M_{7}$ generations following gamma ray treatment of wheat variety San Pastore

Best fitting model

\begin{tabular}{|c|c|c|c|c|c|c|c|}
\hline Treatment & Generation & $E_{1}$ & $E_{2}$ & $E_{3}$ & $E_{4}$ & $D$ & $x_{\text {(d.f.) }}^{2}$ \\
\hline \multicolumn{8}{|c|}{ (i) Plant height } \\
\hline \multirow[t]{2}{*}{$10 \mathrm{kr}$} & $\begin{array}{l}\mathbf{M}_{s} \\
\mathbf{M}_{6}\end{array}$ & $\begin{array}{l}22.37 \pm 1.05 \\
53.73 \pm 19.05\end{array}$ & $8 \cdot 32 \pm 1 \cdot 79$ & & & & \multirow{2}{*}{$\begin{array}{l}0.99(3) \\
5.58(7) \\
6.27(7)\end{array}$} \\
\hline & $\mathbf{M}_{7}^{6}$ & $12 \cdot 30 \pm 0.49$ & $1.79 \pm 0.40$ & & $1.76 \pm 0.56$ & & \\
\hline \multirow[t]{2}{*}{$15 \mathrm{kr}$} & $\mathrm{M}_{5}$ (a) & $\begin{array}{l}23.31 \pm 1.10 \\
21.44 \pm 1.69\end{array}$ & $\begin{array}{r}11.07 \pm 2.27 \\
6.50 \pm 3.16\end{array}$ & \multirow[b]{2}{*}{$4.98 \pm 1.56$} & & $77 \cdot 33 \pm 53 \cdot 49+$ & \multirow{2}{*}{$\begin{array}{l}3.56(3) \\
2.26(2) \\
4.44(5) \\
9 \cdot 60(6)\end{array}$} \\
\hline & $\begin{array}{l}\mathbf{M}_{6} \\
\mathbf{M}_{7}\end{array}$ & $\begin{array}{l}13.55 \pm 0.73 \\
11.82 \pm 0.52\end{array}$ & $\begin{array}{l}2.92 \pm 0.90 \\
2.23 \pm 0.62\end{array}$ & & $2 \cdot 09 \pm 1 \cdot 21$ & $93 \cdot 11 \pm 32 \cdot 01$ & \\
\hline \multirow[t]{2}{*}{$20 \mathrm{kr}$} & $\begin{array}{l}\mathbf{M}_{5} \\
\mathbf{M}_{6}\end{array}$ & $\begin{array}{l}22.72 \pm 1.69 \\
14.26 \pm 0.77\end{array}$ & $\begin{array}{l}4.54 \pm 2.67 \\
1.38 \pm 0.66\end{array}$ & \multirow{2}{*}{$6.09 \pm 1.61$} & & $114 \cdot 80 \pm 50 \cdot 32$ & \multirow{2}{*}{$\begin{array}{l}1.28(2) \\
7 \cdot 78(5) \\
3.43(6)\end{array}$} \\
\hline & $\mathbf{M}_{7}$ & $11.54 \pm 0.50$ & $1.67 \pm 0.60$ & & $2 \cdot 17 \pm 0 \cdot 90$ & $99 \cdot 31 \pm 27 \cdot 53$ & \\
\hline \multicolumn{8}{|c|}{ (ii) Kernel number } \\
\hline \multirow[t]{2}{*}{$10 \mathrm{kr}$} & $\begin{array}{l}M_{5}(\mathrm{a}) \\
M_{6 \ddagger}\end{array}$ & $\begin{array}{r}96.78 \pm 4.56 \\
95.95 \pm 4.91 \\
125.50 \pm 44.35\end{array}$ & $7 \cdot 34 \pm 2 \cdot 92$ & & & \multirow[t]{2}{*}{$95 \cdot 20 \pm 45 \cdot 94$} & \multirow{2}{*}{$\begin{array}{l}2 \cdot 24(3) \\
6 \cdot 38(3) \\
4 \cdot 51(7) \\
8 \cdot 65(7)\end{array}$} \\
\hline & $\mathbf{M}_{7}^{0+}$ & $74.91 \pm 2.96$ & $8 \cdot 79 \pm 2 \cdot 22$ & & $7 \cdot 18 \pm 2 \cdot 61$ & & \\
\hline \multirow[t]{2}{*}{$15 \mathrm{kr}$} & $\mathbf{M}_{5}$ & $\begin{array}{r}88.68 \pm 4.18 \\
124.30 \pm 44.03\end{array}$ & $11 \cdot 00 \pm 3 \cdot 39$ & & & & \multirow{2}{*}{$\begin{array}{l}7 \cdot 43(3) \\
3 \cdot 19(7) \\
8 \cdot 38(6)\end{array}$} \\
\hline & $\mathbf{M}_{7}^{0+}$ & $74.86 \pm 3.01$ & $4 \cdot 46 \pm 2 \cdot 01$ & & $11 \cdot 17 \pm 5 \cdot 09$ & $125 \cdot 10 \pm 68 \cdot 04$ & \\
\hline \multirow[t]{2}{*}{$20 \mathrm{kr}$} & $\begin{array}{l}\mathbf{M}_{5}(\mathrm{a}) \\
\text { (b) } \\
\mathbf{M}_{6} \ddagger\end{array}$ & $\begin{array}{r}100 \cdot 30 \pm 4 \cdot 73 \\
96 \cdot 64 \pm 5.22 \\
120 \cdot 60 \pm 42 \cdot 55\end{array}$ & & & & $220 \cdot 20 \pm 68 \cdot 83$ & \multirow{2}{*}{$\begin{array}{r}0.82(3) \\
5.39(3) \\
3.60(7) \\
8.80(7) \\
12.61(7)\end{array}$} \\
\hline & $\begin{array}{l}\mathbf{M}_{7} \text { (a) } \\
\text { (b) }\end{array}$ & $\begin{array}{l}78.55 \pm 3.10 \\
77.87 \pm 3.11\end{array}$ & $\begin{array}{r}10.53 \pm 2.46 \\
9.69 \pm 2.77\end{array}$ & & $9 \cdot 23 \pm 3 \cdot 15$ & $113 \cdot 70 \pm 41 \cdot 80$ & \\
\hline
\end{tabular}

TABLE 5

Model fitting for the variation in plant height and kernel number in the $M_{5}, M_{6}$ and $M_{7}$ generations following gamma ray treatment of wheat variety Bankut-1205

Best fitting model

\begin{tabular}{|c|c|c|c|c|c|c|c|}
\hline Treatment & Generation & $E_{1}$ & $E_{2}$ & $E_{3}$ & $E_{4}$ & D & $x_{(\mathrm{d} \cdot \mathrm{f} .)}^{2}$ \\
\hline \multicolumn{8}{|c|}{ (i) Plant height } \\
\hline $10 \mathrm{kr}$ & $\begin{array}{l}\mathbf{M}_{5} \\
\mathbf{M}_{7}\end{array}$ & $\begin{array}{l}52.51 \pm 3.23 \\
14.18 \pm 0.58\end{array}$ & $4.09 \pm 0.84$ & $4 \cdot 73 \pm 1 \cdot 51$ & $7 \cdot 10 \pm 2 \cdot 44$ & $216 \cdot 70 \pm 54 \cdot 93$ & $\begin{array}{l}6 \cdot 65(3) \\
8 \cdot 74(6)\end{array}$ \\
\hline $15 \mathrm{kr}$ & $\begin{array}{l}\mathbf{M}_{6} \\
\mathbf{M}_{7}\end{array}$ & $\begin{array}{l}27.08 \pm 1.75 \\
16.02 \pm 0.68\end{array}$ & $2 \cdot 25 \pm 0 \cdot 67$ & $1.80 \pm 0.87$ & $5.58 \pm 1.73$ & $80.55 \pm 24.90$ & $\begin{array}{r}8 \cdot 20(6) \\
13 \cdot 37(6) \dagger\end{array}$ \\
\hline $20 \mathrm{kr}$ & $\mathbf{M}_{7}$ & $15 \cdot 42 \pm 0.70$ & $2.99 \pm 0.81$ & $1.54 \pm 0.97 \dagger$ & $5 \cdot 50 \pm 1 \cdot 86$ & & $13.57(6) \dagger$ \\
\hline \multicolumn{8}{|c|}{ (ii) Kernel number } \\
\hline $\begin{array}{l}15 \mathrm{kr} \\
20 \mathrm{kr}\end{array}$ & $\begin{array}{l}M_{6} \\
M_{7}(\mathrm{a}) \\
\mathbf{M}_{6}(\mathrm{~b}) \\
\mathbf{M}_{7} \text { (a) } \\
\mathbf{M}_{6}{ }^{(\mathrm{b})} \\
\mathbf{M}_{7} \text { (a) } \\
\text { (b) }\end{array}$ & $\begin{array}{l}39 \cdot 36 \pm 2 \cdot 81 \\
52 \cdot 53 \pm 2 \cdot 17 \\
54 \cdot 10 \pm 2 \cdot 12 \\
45 \cdot 28 \pm 2 \cdot 87 \\
49 \cdot 35 \pm 2 \cdot 38 \\
49 \cdot 28 \pm 2 \cdot 35 \\
37 \cdot 60 \pm 2 \cdot 47 \\
50 \cdot 61 \pm 2 \cdot 38 \\
51 \cdot 61 \pm 2 \cdot 37\end{array}$ & $\begin{array}{l}2.52 \pm 1 \cdot 33 \\
2.90 \pm 2 \cdot 19 \dagger \\
2.48 \pm 1 \cdot 62 \dagger\end{array}$ & & & $\begin{array}{c}144 \cdot 80 \pm 64 \cdot 06 \\
54 \cdot 45 \pm 20 \cdot 67 \\
69 \cdot 17 \pm 20 \cdot 57 \\
82 \cdot 78 \pm 31 \cdot 08 \\
283 \cdot 90 \pm 167 \cdot 63 \\
439 \cdot 90 \pm 108 \cdot 78 \\
72 \cdot 29 \pm 31.95 \\
164 \cdot 00 \pm 55 \cdot 11 \\
206 \cdot 10 \pm 55 \cdot 29\end{array}$ & $\begin{array}{r}3.20(6) \\
3.44(7) \\
7.94(8) \\
1.49(6) \\
11.73(7) \\
14.41(8) \\
6.10(6) \\
3.97(7) \\
6.89(8)\end{array}$ \\
\hline
\end{tabular}

the only justification for including $E_{2}$ is that in the hierarchical analyses of variance of the controls this component was significant $(P<0 \cdot 05)$.

In the remaining cases, all for San Pastore, the ambiguity is more critical; we are faced with two alternative models both of which are adequate but only one of which has a significant additive genetic component. The latter can either be omitted without failure of the residual model or it can be replaced by an additional environmental component $\left(E_{2}\right.$ or $\left.E_{4}\right)$. Further- 
more this substitution of an $E$ for the $D$ component reduced the $\chi^{2}$ value and these $E$ components are significant in the hierarchical analysis of variance of the controls $(\mathrm{P}<0.05)$. For these cases, therefore, the evidence for a genetical component of variation is not strong.

The $M_{5}, M_{6}$ and $M_{7}$ generation means for the treatments and controls are given by Borojević and Borojević (1969). For the five combinations for which there were significant deviations between the means of the treatments and the corresponding controls, models have been fitted. To do this it is necessary to combine in a weighted least squares analysis the means of the $\mathrm{M}_{5}, \mathrm{M}_{6}$ and $\mathrm{M}_{7}$ generations, each of which was raised in a different season. Differences between generations which in our models are formally ascribable to dominance (table 2) will, therefore, be confounded with seasonal differences. If the latter are additive, however, they can be removed by working with the deviations from the means of the controls in the same season. Any non-additive effects of seasons, however, will still be confounded with the dominance effects or may even lead to the failure of the additive, dominance model.

\section{TABLE 6}

Model fitting on means of plant height and grain number recorded in $M_{5}, M_{6}$ and $M_{7}$ generations of gamma ray treated Bankut-1205 and San Pastore wheat varieties

\begin{tabular}{|c|c|c|c|c|c|}
\hline \multirow[b]{2}{*}{ Parameter } & \multicolumn{2}{|c|}{$\begin{array}{c}\text { Plant height } \\
\text { (variety, Bankut-1205) }\end{array}$} & \multicolumn{3}{|c|}{$\begin{array}{c}\text { Kernel number } \\
\text { (variety, S. Pastore) }\end{array}$} \\
\hline & $15 \mathrm{kr}$ & $20 \mathrm{kr}$ & $10 \mathrm{kr}$ & $15 \mathrm{kr}$ & $20 \mathrm{kr}$ \\
\hline$[d]$ & $\begin{aligned} & 2 \cdot 54^{*} \\
\pm & 0 \cdot 38\end{aligned}$ & 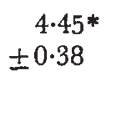 & $\begin{array}{c}2.20^{*} \\
\pm 0.54 \\
63.81^{*} \\
\pm 17.56\end{array}$ & $\begin{array}{c}2 \cdot 12^{*} \\
\pm 0 \cdot 54 \\
59 \cdot 51^{*} \\
\pm 17 \cdot 91\end{array}$ & $\begin{array}{c}6.67^{*} \\
\pm 0.52 \\
121.00^{*} \\
\pm 17.59\end{array}$ \\
\hline$\chi_{(d . f .)}^{2}$ & $2 \cdot 70(2)$ & $4 \cdot 90(2)$ & $3 \cdot 39(1)$ & $0.47(1)$ & $2 \cdot 40(1)$ \\
\hline
\end{tabular}

The results of fitting the additive, dominance model to the deviations are summarised in table 6 for those combinations where the deviations are significant. In all cases this simple model is adequate. Indeed for plant height in Bankut-1205 a model consisting of $[d]$ alone is adequate. This inability to detect a dominance contribution could merely reflect the high degree of homozygosity in the $\mathrm{M}_{5}$ to $\mathrm{M}_{3}$ generations. For kernel number in San Pastore, on the other hand, the estimates of $[h]$ are relatively very high and significant, although they have large standard errors. These estimates could, of course, reflect non-additive effects of seasonal differences as well as dominance as we have already noted. Equally, however, they could reflect the effects of dispersion which will reduce the magnitude of $[d]$ relative to $[h]$, particularly if the dominance is unidirectional.

\section{Discussion}

In this paper we have shown that quantitative data from mutation breeding experiments can be simply parameterised to produce a description of induced variation which has predictive value. This has been achieved 
by (i) considering the means, variances and covariances that can be generated if a hierarchically structured selfing series is initiated from each individual of the $\mathrm{M}_{1}$, and (ii) by omitting from the analyses all rank 0 statistics. It provides the simplest possible tests of the adequacy of biometrical genetical models of the means and variances and estimates of all the parameters required to measure the induced variation and for predicting the long-term outcome of the inbreeding programme.

This approach explicitly confines attention to the variation arising from the segregation of the induced heterozygosity in the $M_{1}$ plants and treats the differences between $M_{1}$ plants in this respect as differences between replicates. This avoids the only controversial aspect of modelling induced variation, namely, having to make assumptions about the independence or kind of dependence of mutational events. By making such assumptions models for the rank 0 variances corresponding with those for other statistics can be produced. But even with the most restrictive assumptions two additional genetical parameters are required to carry out a combined analysis of statistics of all ranks. The information gained from including the rank 0 statistics is, therefore, partially or wholly offset in estimating the additional parameters and the additional assumptions on which they are defined become a possible cause of failure of the model. If, however, the model fits, these additional parameters permit a description of the variation induced between as well as within $\mathbf{M}_{1}$ plants but they are less informative about the underlying gene action than the parameters obtainable from statistics of lesser rank. Ideally, of course, one would wish to test the goodness-of-fit and estimate the parameters of a model which is based on the least restrictive assumptions even though this will add yet one further genetical parameter and hence require at least one more observed statistic.

Other considerations, however, enter into the availability of statistics and hence the complexity of the model that in practice can be tested. In the early generations the proportion of rank 0 statistics is high, and any analysis that excluded them would be making very inefficient use of the data. On the other hand, because of physiological damage in the $M_{1}$ generation any statistic which depends upon $\mathrm{M}_{1}$ plants directly should not be used, e.g. $V_{0 M_{1}}, W_{0 M_{12}}, W_{0 M_{13}}$ etc. Furthermore, to provide sufficient statistics to fit a model in the early generations statistics from two or more successive generations would have to be combined. Since successive generations are usually raised in successive seasons any interaction between genotype and seasonal differences would lead to failure of the model; a complication which applies equally if the remaining narent-offsnring covariances $\left(W_{1}, W_{2}\right.$ etc) are used. This complication can be avorded by raising further samples of all earlier generations in every season. But this completely negates the value of including rank 0 statistics in the analysis in order to improve the rate of utilisation of the data from the early generations. A preferable way out of these difficulties, therefore, from the $M_{3}$ generation onwards, is to follow the procedures and analyses described in this paper.

The illustrative examples of these analyses are relatively disappointing but no more so than could have been predicted from the published hierarchical analyses of variances. In only a minority of cases could we find a satisfactory model and at the same time demonstrate a significant genetical component of variation. From the distribution of some of these failures they can only be a combination of a small genetical component and relatively 
large sampling errors. Others, however, are more systematic in their occurrence and the largest, single, identifiable cause is the significances among the higher ranking variances in the control material. Examples are plant height in the $M_{7}$ generation of Bankut-1205 for all three doses of irradiation and plant height in the $\mathbf{M}_{6}$ generation of San Pastore for all three doses of irradiation. These must involve a failure in the experimental design or of the experimental material which we can attempt to accommodate in our analyses only by introducing additional environmental components $E_{3}$ and $E_{4}$.

The analyses of the generation means while only marginally more successful nevertheless give a consistent picture at a number of levels. For example, $[d]$, which measures the difference in frequency of mutations of increasing alleles to decreasing alleles and of decreasing alleles to increasing alleles is significantly positive for all three doses for kernel number in San Pastore and for the top two doses for plant height in Bankut-1205. Furthermore, where $[d]$ is significant it is significantly greater in magnitude at the highest dose of irradiation than at the lower dose.

Since all significant estimates of $[d]$ are positive, more decreasing alleles have mutated to increasing alleles than the reverse. Either therefore, the decreasing alleles have higher rates of mutation or, as seems more likely, San Pastore and Bankut-1205 have more decreasing than increasing alleles for plant height and kernel number. But what is clear is that pure-breeding lines with higher scores than the original pair of lines can be obtained for both characters. Although the significant estimates of $[h]$ must be viewed with caution they too are consistently positive implying that more increasing than decreasing alleles are dominant.

In this paper we have considered how induced variation may be analysed using the model fitting procedures of biometrical genetics within the context of the information that is generated during the early stages of observing and fixing the variation following the mutagenic treatment. This information is far from ideal for model fitting and it is particularly unsatisfactory for detecting and estimating non-additive sources of variation. Where, therefore, a primary objective is an understanding of the nature of the induced variation and its genetical architecture one of the many mating systems specially designed for this purpose, such as test crosses (Kearsey and Jinks, 1968 and Virk and Jinks, 1977) or diallel sets of crosses (see Mather and Jinks, 1971, and Dickinson and Jinks, 1956) should be used either on early generation material such as the $M_{2}$ or on inbreds derived from it (Lawrence, 1968).

Acknowledgements.-These are due to the Royal Commission for the Exhibition of 1851 for financial support to D. S. V. during these investigations.

\section{REFERENGES}

AASTVEIT, K., AND GAUL, H. 1967. Variation and selection of micro-mutants. Radiation Botany, 7, 353-361.

BOROJEVIĆ, K., AND BOROJEvić, s. 1969. Stabilization of induced genetic variability in irradiated populations of vulgare wheat. IAEA/FAO Symp. Induced mutations in plants, 399-432.

DIGKINSON, A. G., AND JINKs, J. L. 1956. A generalised analysis of diallel crosses. Genetics, 41, 65-78.

GAUL, H. 1965. The concept of macro- and micro-mutations and results on induced micromutations in barley. Radiation Botany, 5 (suppl.), 407-428. 
JINKs, J. L., AND POONI, H. s. 1976. Predicting the properties of recombinant inbred lines derived by single seed descent. Heredity, 36 (2), 253-266.

KAO, K. N., HU, C. H., CHANG, W. T., AND OKA, H. r. 1960. A biometrical-genetic study of irradiated populations in rice; genetic variances due to different doses of X-rays. Bot. Bull. Acad. Sin., 1, 101-108.

KEARSEY, M. J., AND JINKs, J. L. 1968. A general method of detecting additive dominance and epistatic variation for metrical traits. I. Theory. Heredity, 22, 97-103.

LAWRENGE, c. w. 1968. Radiation-induced polygenic mutation in Arabidopsis thaliana II. Analysis of lines selected for flowering time. Heredity, 23, 573-589.

MATHER, K., AND Jinks, J. L. 1971. Biometrical Genetics, 2nd edition. Chapman and Hall, London.

viRK, D. S., AND JINKs, J. L. 1977. The consequences of using inadequate testers in the simplified triple test-cross. Heredity, 38, 237-251. 